Trauma Berufskrankh 2008 10 [Suppl 3]:311-315

DOI 10.1007/s10039-008-1413-x

Online publiziert: 17. Mai 2008

(c) Springer Medizin Verlag 2008

\author{
M. Gutbier \\ BG-Unfallklinik, Ludwigshafen
}

\title{
Zusammenhangstrennung der Rotatorenmanschette
}

\author{
Begleit- und Nachbehandlung
}

Anders als bei stabilen Osteosynthesen benötigt der Physiotherapeut sowohl bei der konservativen als auch der postoperativen Behandlung der Rotatorenmanschette ein besonderes Fingerspitzengefühl.

Bekanntlich ist bei degenerativen Veränderungen die Zusammenhangstrennung der Rotatorenmanschette nach vielen Mikrotraumen im Sport oder im Berufsleben bei Überkopfarbeitern der so genannte letzte Tropfen, der das Fass zum Überlaufen bringt. Da die Indikation zur Operation nur bei frischen Traumen oder degenerativen Veränderungen mit ausreichend konservativer Vorbehandlung gegeben ist, kommt der konservativen, in der Regel ambulanten physiotherapeutischen Behandlung entscheidende Bedeutung zu. Sie wird im ersten Teil dieses Beitrags abgehandelt. Im 2. Teil wird das Prozedere der physiotherapeutischen Therapie nach Operation der Rotatorenmanschette vorgestellt.

\section{Konservative physio- therapeutische Therapie}

Prinzipiell muss klar sein, dass ein Schaden der Rotatorenmanschette an der Sehnenplatte durch keine der vielen zur Verfügung stehenden physiotherapeutischen Maßnahmen behoben werden kann.

Bei Patienten, die wegen einer Zusammenhangstrennung der Rotatorenmanschette zur physiotherapeutischen Behandlung kommen, ist das Schultergelenk im Gilchrist-Verband (- Abb. 1) oder in der Bronner-Schlinge ( $\bullet$ Abb. 2) entlastet. Zunächst steht das Problem der Schmerzsymptomatik im Vordergrund.

\section{Befunderhebung}

$\mathrm{Zu}$ Therapiebeginn werden der physiotherapeutische Befund oder der funktionelle Status erhoben sowie Muskeltests durchgeführt, die Aufschluss über die betroffenen geschädigten Strukturen geben:

- Schmerz bei Widerstand gegen die Abduktion aus der Nullstellung im Schultergelenk bedeutet, dass die Supraspinatussehne verletzt ist.

- Mit dem Test bei $90^{\circ}$ Abduktion, 30 transversaler Flexion und Innenrotation kann abgeklärt werden, ob der Supraspinatusmuskel betroffen ist.

- Ein „painful arc“, d. h. die Schmerzauslösung bei aktiver Abduktion im Schultergelenk zwischen 60 und $90^{\circ}$, kann sowohl durch eine Schädigung der Supraspinatussehne als auch der Bursa subacromialis bedingt sein.

- Die Mm. infraspinatus und teres minor werden mit Außenrotation gegen Widerstand getestet, der M. subscapularis mit dem so genannten Schürzentest (Innenrotation) oder Napoleon-Zeichen.

Es empfiehlt sich, zu Beginn der Behandlungsserie, die über 4-6 Wochen verläuft, eine Schulterfunktionsbeurteilung zu erfassen, um den Therapieerfolg bei einem Retest am Behandlungsende werten zu können (DASH-Fragebogen). Im Constant-Bewertungsschema werden neben Schmerz, Bewegungsumfang und
Kraft auch Parameter der Teilhabe in Freizeit, Sport und Beruf berücksichtigt (• Tab. 1).

\section{Ziele der konservativen Behandlung}

Sie liegen primär im

- Reduzieren des Schmerzes,

- Erreichen der bestmöglichen Beweglichkeit und

- Wiedererlangen der Alltagsaktivitäten, gleichbedeutend der Teilhabe an Beruf, Hobby und Freizeitaktivitäten.

\section{Methoden}

Unsere Maßnahmen zur Schmerzreduktion beinhalten die Wiederherstellung eines normalen Tonus der Muskulatur durch Eis- und Wärmebehandlung, Elektrotherapie, Massagen und entlastete Bewegungen im Schlingentisch. Im Bewegungsbad nutzen wir den Auftrieb des Wassers zur Entlastung und später auch den Wasserwiderstand zur Kräftigung der Muskulatur.

Tab. 1 Schulterfunktionsbeurteilung mit Hilfe des Constant-Bewertungsschemas

\begin{tabular}{|ll}
\hline Funktion & Punkte \\
Schmerz & $0-15$ \\
\hline Alltagsaktivitäten & $0-20$ \\
\hline Bewegungsumfang & $0-40$ \\
\hline Kraft & $0-25$ \\
\hline Summe (maximal) & 100 \\
\hline
\end{tabular}




\section{Verletzungen und Verletzungsfolgen am Schultergelenk}
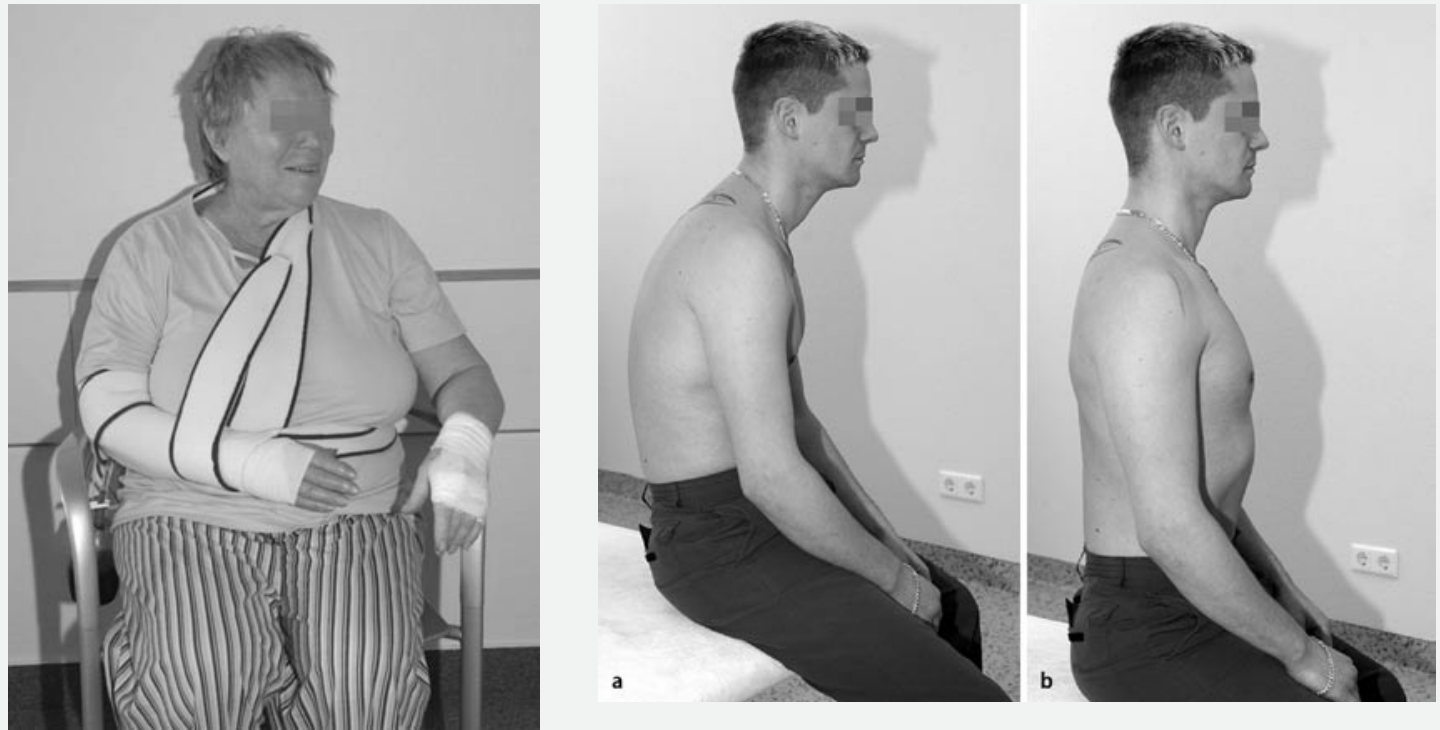

Abb. $3<$ Haltungsschulung, a saloppe, b aufrechte Haltung

Abb. $1 \Delta$ Gilchrist-Verband
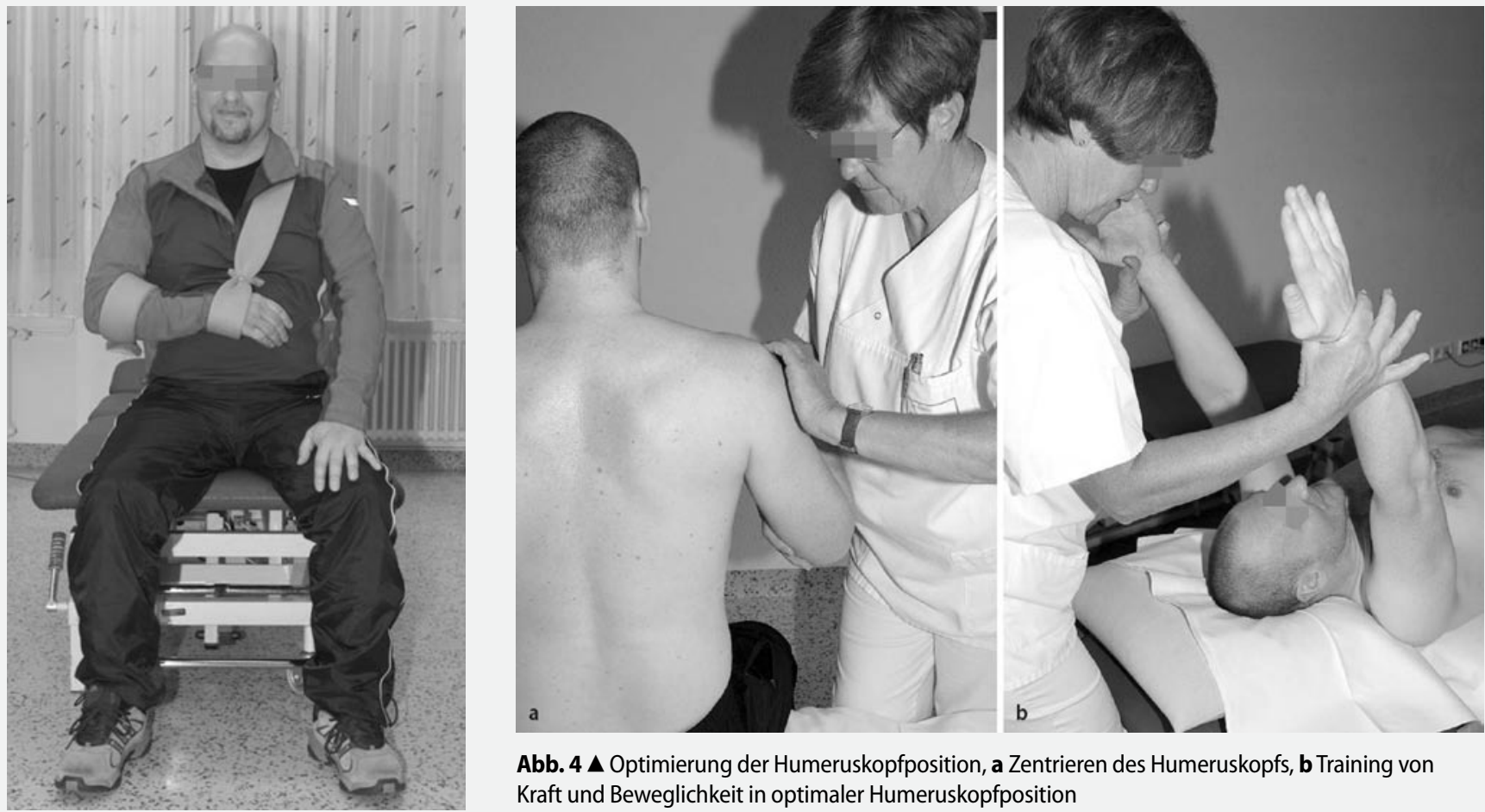

Abb. 4 \ Optimierung der Humeruskopfposition, a Zentrieren des Humeruskopfs, b Training von Kraft und Beweglichkeit in optimaler Humeruskopfposition

Abb. $2 \Delta$ Bronner-Schlinge

Entspannungstherapie und Weichteiltechniken z. B. aus der manuellen Therapie oder der funktionellen Bewegungslehre Klein-Vogelbach unterstützen die Schmerzlinderung. Konnte der Schmerz mit den physiotherapeutischen Maßnahmen deutlich reduziert werden, steht die Behandlung der Bewegungseinschränkung im Vordergrund. Eine Verbesserung der Beweglichkeit muss Hand in Hand mit der Kräftigung der Schultermuskulatur einhergehen. Insbesondere, wenn be- stimmte Strukturen irreparabel sind, müssen andere Muskeln kompensatorisch die Aufgabe der Stabilisierung übernehmen und entsprechend trainiert werden.

Haltungsschulung ist inzwischen ein „abgedroschener" Begriff, aber wie aus - Abb. 3 ersichtlich, für die Stellung des Schultergelenks von zentraler Bedeutung. Eine saloppe Haltung (• Abb. 3a) mit Protraktion der Schulter bedeutet für das Schultergelenk eine Verengung des subakromialen Raums und eine wesentlich erhöhte Kapselspannung, da der Humeruskopf nach ventral geglitten und somit nicht mehr zentriert ist. Mit der Haltungsschulung ist auch die Wahrnehmungsschulung ein bedeutendes Element der Behandlung (- Abb. 3b).

Der Patient soll in der Lage sein, die korrekte Position seines Humeruskopfs zu spüren (- Abb. 4a). Dazu sind grundsätzlich Anleitungen zum regelmäßigen Eigentraining erforderlich, damit die Behandlung eine Chance auf einen dauer- 


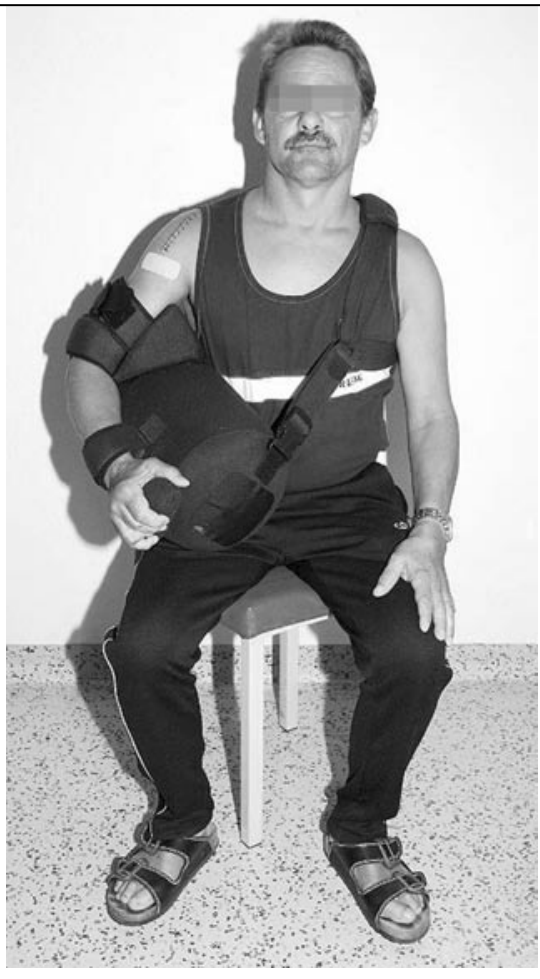

Abb. $5 \Delta$ Schulterabduktionskissen (SAK)

haften Erfolg hat. Somit ist unser wichtigstes Ziel, nach der Schmerzbehandlung die Beweglichkeit und die Kraft in einer optimalen Position des Humeruskopfs in seiner kleinen Pfanne wiederherzustellen

(• Abb. 4b).

\section{Physiotherapeutische Therapie nach Operation der Rotatorenmanschette}

Dem Patienten wird bereits im OP ein Schulterabduktionskissen (SAK) angelegt (- Abb.5).

Am 1. postoperativen Tag wird dem Patienten gezeigt, wie er über die nicht betroffene Seite aus dem Bett aufsteht, damit ungewollte Abstützaktivitäten vermieden werden. Um die bereits beschriebenen Fehlhaltungen zu vermeiden, wird eine Haltungsstabilisation, die dem Patienten das Empfinden für die Stellung seines Humeruskopfs übermittelt, vorgenommen. Aus dem SAK sind Umkehrbewegungen des Unterarms in Flexion/Extension und Supination/Pronation im Ellenbogengelenk erlaubt.

$\mathrm{Ab}$ 2. Tag postoperativ ist nur die passive Abduktion und Flexion zwischen 60 und $90^{\circ}$ erlaubt. Diese Bewegungen werden aus einer entsprechenden Lagerung
Trauma Berufskrankh 2008 · 10 [Suppl 3]:311-315 DOI 10.1007/s10039-008-1413-x

(c) Springer Medizin Verlag 2008

\section{Gutbier}

\section{Zusammenhangstrennung der Rotatorenmanschette.} Begleit- und Nachbehandlung

\section{Zusammenfassung}

Da eine Operation bei Zusammenhangstrennung der Rotatorenmanschette nur bei frischen Traumen oder degenerativen Veränderungen mit ausreichend konservativer Vorbehandlung indiziert ist, kommt der physiotherapeutischen Behandlung, die den Schaden selbst natürlich nicht beseitigen kann, entscheidende Bedeutung zu. An ihrem Beginn steht die physiotherapeutische Befunderhebung inklusive Muskeltests. Zudem empfiehlt sich die Erfassung einer Schulterfunktionsbeurteilung. Zunächst steht die Schmerzreduktion im Vordergrund der Therapie. Nach Erreichen dieses primären Therapieziels schließen sich Übungen zur Wiederherstellung der Beweglichkeit und der Kraft in einer optimalen Position des Humeruskopfs in seiner klei-

nen Pfanne an. Im 2. Teil des Beitrags wird das Prozedere nach Operation der Rotatorenmanschette vorgestellt, das sich in einen direkt postoperativen stationären, einen ambulanten und - bei BG-Patienten - einen erneuten stationären Teil gliedert. Sukzessive kann mehr Belastung erlaubt werden, nach $1 / 2$ Jahr etwa sollte die Belastungssteigerung über $25 \mathrm{~kg}$ möglich sein, und "Überkopfsportarten" oder berufliche Tätigkeiten, die ein Arbeiten über Kopf verlangen, können in Angriff genommen werden.

\section{Schlüsselwörter}

Rotatorenmanschette Physiotherapie .

Schmerzreduktion · Beweglichkeit · Kraft

\section{Damage to the structural integrity of the rotator cuff. Treatment and aftercare}

\section{Abstract}

Since surgery in rotator cuff tears is only indicated in the case of fresh trauma or degenerative changes with sufficient prior conservative treatment, the latter assumes particular significance. In this case, naturally even physiotherapy is unable to treat the damage. Treatment begins with a physiotherapeutic assessment of the findings, including muscle tests. An assessment of shoulder function is also recommended. Pain reduction is given priority. Exercises designed to restore mobility and strength with the humeral head in an optimal position in its socket are then carried out. The second part of the article presents the procedure following rotator cuff sur- gery, which is divided into an immediate postoperative in-patient stage and an outpatient stage; for those with employee compensation insurance, a further in-patient stay in hospital comprises a third stage. Weightbearing can be gradually allowed, such that the load increase after 6 months should exceed $25 \mathrm{~kg}$; "overhead" sports or occupational activities which require overhead tasks can be tackled.

\section{Keywords}

Rotator cuff · Physiotherapy - Pain reduction . Mobility $\cdot$ Strength 


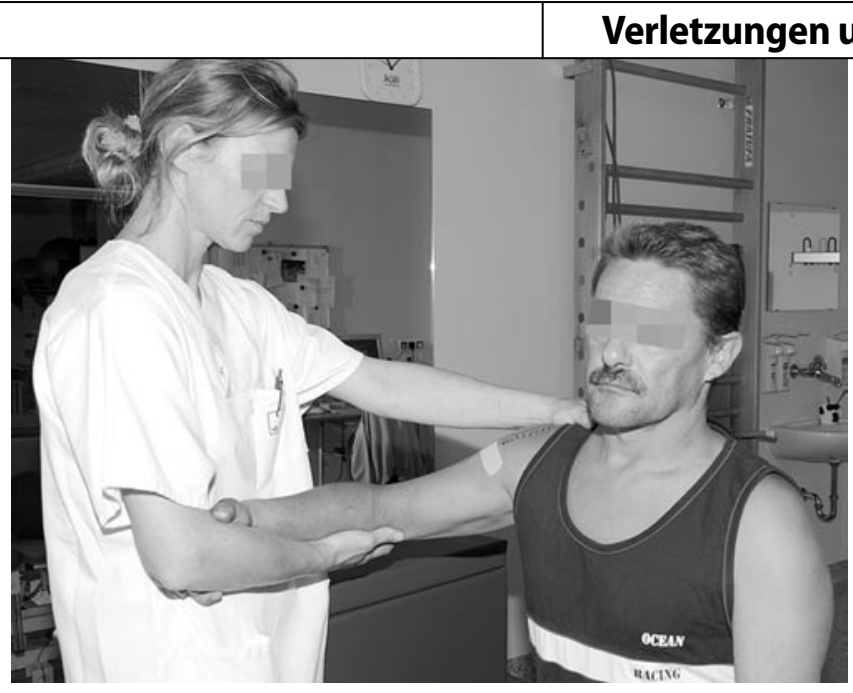

Abb. $6 \Delta$ Lagerung in der ASTE (Ausgangsstellung) Sitz

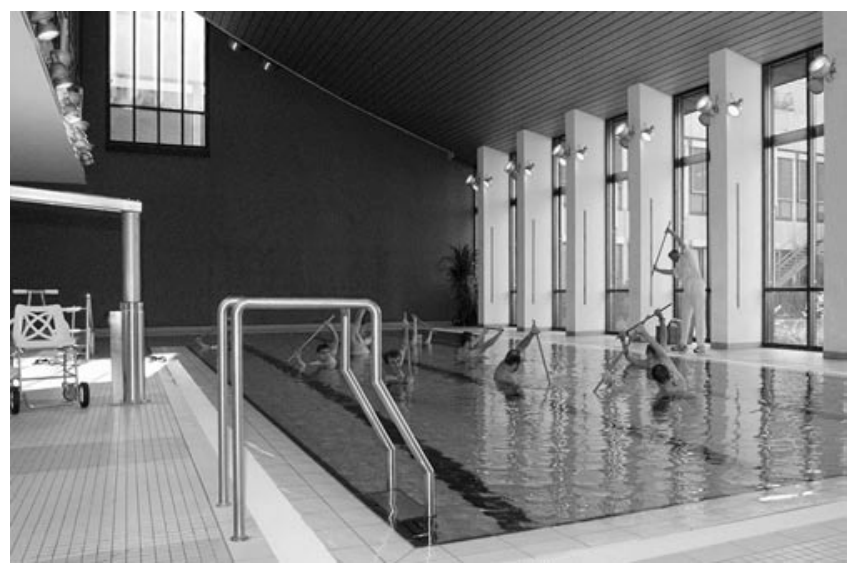

Abb. $8<$ Gruppentherapie im Bewegungsbad

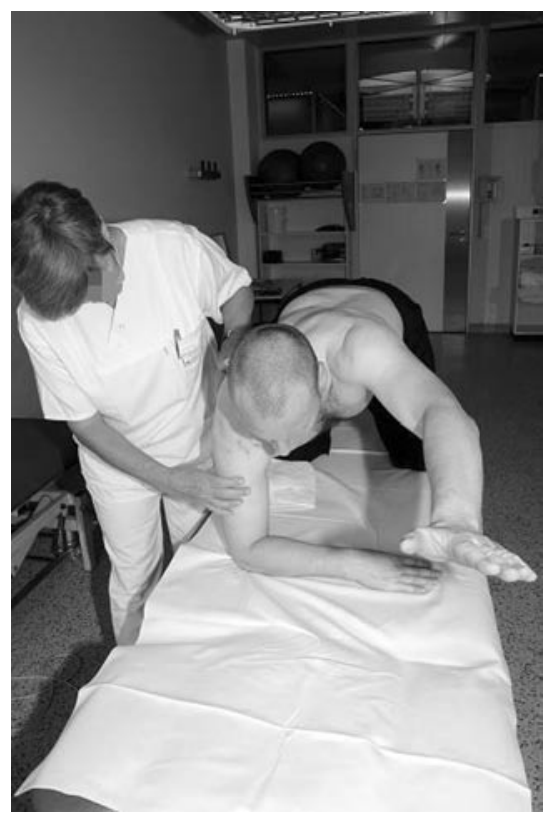

Abb. $9 \Delta$ Stützfunktion

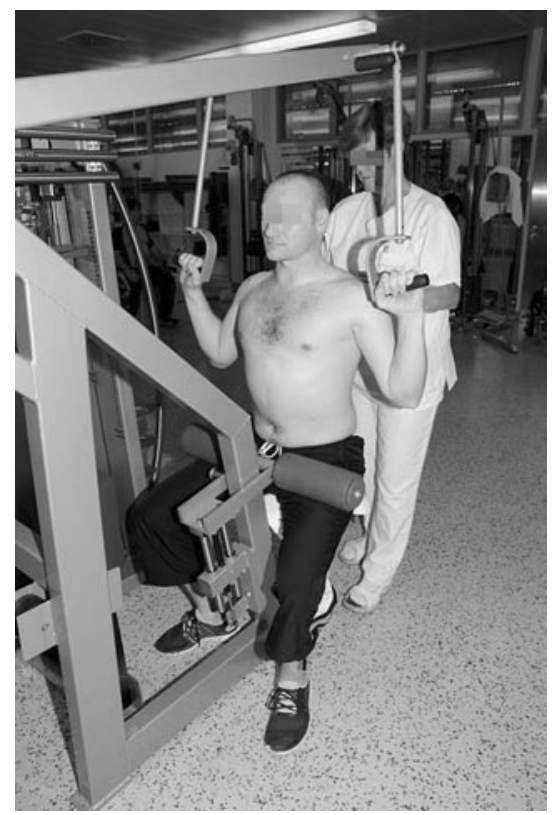

Abb. $10 \Delta$ Medizinische Trainingstherapie

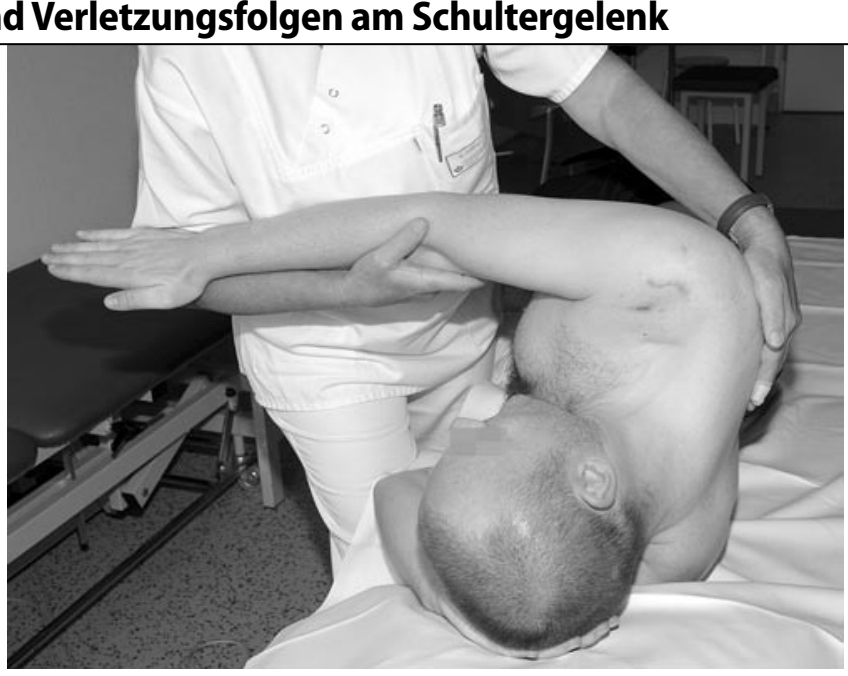

Abb. 7 \ Passive Bewegungen des Schultergürtels

im Sitz oder im Schlingentisch ausgeführt (- Abb. 6).

Für den weiterbehandelnden Physiotherapeuten wird dem Patienten die schriftliche Information mitgegeben, dass die Schulter bis zur 4. Woche postoperativ nur passiv/assistiv zwischen 60 und $90^{\circ}$ in Flexion und Abduktion und bis maximal $20^{\circ}$ in Außenrotation bewegt werden darf. Eine Innenrotation gegen Widerstand (z. B. starker Druck mit der Hand auf das Abduktionskissen) ist nicht erlaubt. Das Hängenlassen des Armes bis zur Nullstellung, also in Adduktion, ist ggf. nur nach Rücksprache mit dem Operateur gestattet. Die Umkehrbewegungen des Unterarms im Ellenbogengelenk sollen weiterhin durchgeführt werden, ebenso die Haltungsschulung, die konsequent wahrnehmungsbezogen instruiert werden muss. Der Schultergürtel darf passiv bewegt werden, so lange die erlaubten Bewegungsausmaße im Schultergelenk eingehalten werden ( $\bullet$ Abb. 7). Dies dient der skapulathorakalen Gleitfähigkeit und der Tonusregulierung für die den Schultergürtel umgebende Muskulatur.

Nach 4 Wochen wird die Lagerung auf dem SAK beendet, und als „ Entwöhnungszwischenschritt“ wird dem Patienten eine Bronner-Schlinge angelegt. Sie besteht aus einem Schaumstoffschlauch, der am proximalen Unterarm befestigt und über den Rücken und die kontralaterale Schulter zum distalen Unterarm geführt wird (• Abb. 2). Nun rückt der Zeitpunkt näher, an dem BG-Patienten erneut zur stationären Rehabilitation kommen. 


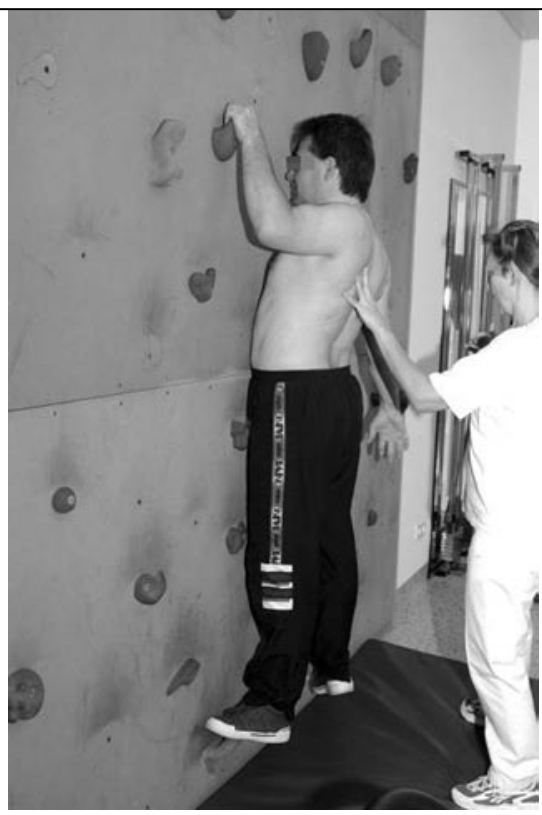

Abb. $11 \Delta$ Therapeutisches Klettern

Neben dem aktuellen Befund sollte jetzt auch die Schulterfunktion schriftlich beurteilt werden, die nun maximal 50 Punkte bzw. Prozent erwarten lässt. Es wird mit vorsichtigen aktiv/assistiven Bewegungen im Schultergelenk begonnen, wobei die Bewegung weiterhin bei fixierter Skapula für Abduktion und Flexion bis $90^{\circ}$ limitiert wird.

Ab der 7. Woche postoperativ besteht Bewegungsstabilität in alle Richtungen ohne Limit, aktive Widerlagerung und widerlagernde Mobilisation sind gegen Widerstände von 5-10 kg erlaubt. Der Armergometer ist jetzt im Rahmen eines Aufwärm- und Ausdauerprogramms indiziert. In der medizinischen Trainingstherapie darf der Patient nun auch in andere Trainingsgeräte, die mit dosierten Gewichten die erlaubte Belastung gewährleisten, eingewiesen werden. Im Bewegungsbad kann er sowohl an Gruppentherapien teilnehmen als auch abhängig von seiner erreichten Beweglichkeit frei schwimmen (• Abb. 8).

$\mathrm{Ab}$ der 12. Woche steigt die Belastbarkeit über 10-25 kg. Jetzt kann die Behandlung bevorzugt in Stützfunktion ausgeführt werden, da dabei die Muskulatur reaktiv besonders in ihrer rotatorischen Verschraubung gefordert wird (• Abb. 9). In der medizinischen Trainingstherapie können Gewichte bis $25 \mathrm{~kg}$ in alle Bewegungsrichtungen, angepasst an die Kraft des $\mathrm{Pa}$ tienten, eingesetzt werden ( $\bullet$ Abb. 10).

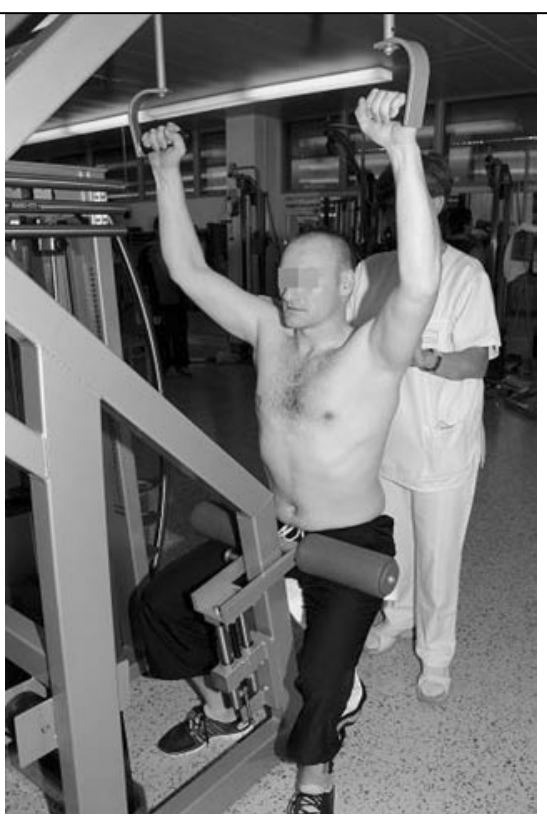

Abb. $12 \Delta$ Belastungssteigerung über $25 \mathrm{~kg}$

Insbesondere der Seilzugapparat erlaubt alle nur denkbaren Bewegungskombinationen. Wichtig ist bei diesem Gerät, dass der Patient sehr gut eingewiesen wird, da sich ansonsten Ausweichbewegungen, die eher schaden als nutzen könnten, einschleichen. Auch mit der Sporttherapie kann ab der 12. Woche postoperativ begonnen werden. In Sport und Spiel wie beim Kegeln, Klettern, Tischtennis, usw. werden erreichtes Bewegungsausmaß und wiedererlangte Kraft funktionell eingesetzt (• Abb. 11).

Nach 6 Monaten postoperativ sollte die Belastungssteigerung über $25 \mathrm{~kg}$ möglich sein, und „Überkopfsportarten“ oder berufliche Tätigkeiten, die ein Arbeiten über Kopf verlangen, können in Angriff genommen werden ( $\bullet$ Abb. 12).

\section{Korrespondenzadresse}

\section{Gutbier}

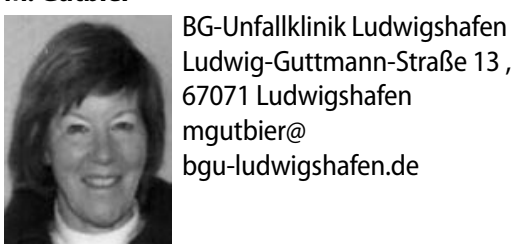

Interessenkonflikt. Der korrespondierende Autor gibt an, dass kein Interessenkonflikt besteht. 